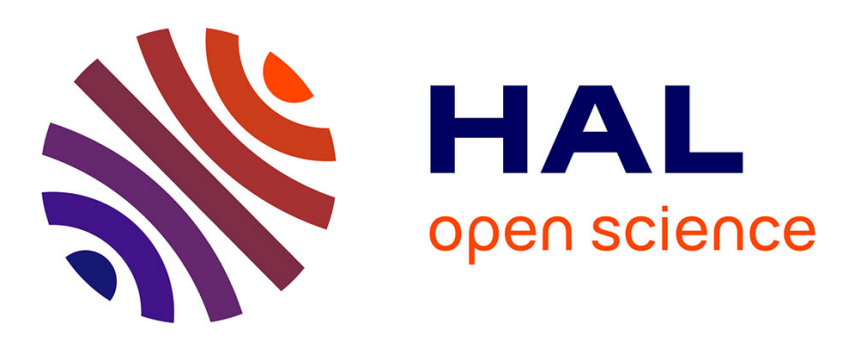

\title{
Light and shadow on the mechanisms of integration site selection in yeast $\mathrm{Ty}$ retrotransposon families
}

\author{
Amandine Bonnet, Pascale Lesage
}

\section{To cite this version:}

Amandine Bonnet, Pascale Lesage. Light and shadow on the mechanisms of integration site selection in yeast Ty retrotransposon families. Current Genetics, 2021, 67 (3), pp.347-357. 10.1007/s00294021-01154-7 . hal-03419297

\section{HAL Id: hal-03419297 \\ https://hal.science/hal-03419297}

Submitted on 22 Nov 2021

HAL is a multi-disciplinary open access archive for the deposit and dissemination of scientific research documents, whether they are published or not. The documents may come from teaching and research institutions in France or abroad, or from public or private research centers.
L'archive ouverte pluridisciplinaire HAL, est destinée au dépôt et à la diffusion de documents scientifiques de niveau recherche, publiés ou non, émanant des établissements d'enseignement et de recherche français ou étrangers, des laboratoires publics ou privés. 
4 Authors

5 Amandine Bonnet and Pascale Lesage

6

\section{Affiliations}

8 INSERM U944, CNRS UMR 7212, Genomes \& Cell Biology of Disease Unit, Institut de

9 Recherche Saint-Louis, Université de Paris, Hôpital Saint-Louis, Paris, France.

10 e-mails: amandine.bonnet@inserm.fr ; pascale.lesage@,inserm.fr

11

12 


\section{Abstract}

14 Transposable elements are ubiquitous in genomes. Their successful expansion depends in part 15 on their sites of integration in their host genome. In Saccharomyces cerevisiae, evolution has 16 selected various strategies to target the five Ty LTR-retrotransposon families into gene-poor 17 regions in a genome, where coding sequences occupy $70 \%$ of the DNA. The integration of 18 Ty1/Ty2/Ty4 and Ty3 occurs upstream and at the transcription start site of the genes 19 transcribed by RNA polymerase III, respectively. Ty5 has completely different integration 20 site preferences, targeting heterochromatin regions. Here, we review the history that led to the 21 identification of the cellular tethering factors that play a major role in anchoring Ty 22 retrotransposons to their preferred sites. We also question the involvement of additional factors in the fine-tuning of the integration site selection, with several studies converging towards an importance of the structure and organization of the chromatin.

Key Words 
Transposable elements (TEs) are mobile repeated DNA sequences present in almost all 31 organisms, where they represent a significant, albeit variable, proportion of their genomes. Although Britten and Davidson had already suggested in the late 1960s that TEs could drive the evolution of gene regulatory networks (Britten and Davidson, 1969), these have long been considered as junk and selfish pieces of DNA that colonize host organisms and threaten genome integrity. TEs are indeed powerful mutagens that can affect host genomes upon insertion of a new copy or by recombination between existing copies leading to large-scale genomic rearrangements. New TE integrations can have multiple short-term deleterious effects on genetic integrity and are implicated in various human diseases (Burns, 2017; Hancks and Kazazian, 2012). Yet they also play a fundamental role in shaping genomes throughout evolution (Chuong et al., 2016). Several iconic examples in plants, insects and mammals show that TEs can trigger phenotypic diversity and initiate new adaptive phenotypes through various genetic and epigenetic mechanisms (Chuong et al., 2016).

The consequences of a new TE copy for host biology and genome integrity is closely linked to its integration site. Broad patterns of non-random distribution have been recorded in many organisms indicating that TEs accumulate in specific regions of their host genome (for review (Sultana et al., 2017)). This genomic distribution of TEs results from the balance between two forces. On the one hand, well-known post-integration natural selection processes remove strongly deleterious new insertions and perpetuate beneficial ones. On the other hand, an active process targets most TEs to specific regions of the genome, as revealed by highthroughput profiling of de novo TE integration sites by next-generation sequencing. TEs exhibit a high level of diversity for their integration site preferences, which depend on a wide range of genomic features, from specific nucleotide sequences to 3D chromosomal organization. For a detailed description of the diversity of TE targeting, several studies can be consulted (Cheung et al., 2018; Cosby et al., 2019; Levin and Moran, 2011; Spaller et al., 2016; Sultana et al., 2017).

Retrotransposons, which replicate through an RNA intermediate, are the main active TEs in mammals and yeast and are structurally and functionally related to retroviruses. Investigation of the integration preferences of several retrotransposons and retroviruses has undoubtedly been instrumental in understanding the molecular basis of TE integration selectivity (Bushman, 2003; Kvaratskhelia et al., 2014; Sultana et al., 2017). Taking advantage of the 
genetically easy-to-manipulate budding yeast Saccharomyces cerevisiae, the integration of the yeast Ty retrotransposons has been especially well studied, establishing some fundamentals of TE integration targeting.

\section{Ty retrotransposons have acquired different integration strategies to avoid genes and} propagate

TE insertion patterns depend on complex interactions between the TE integration machineries and their host cellular environment. Integration preferences result from two opposing constraints that allow the subsequent expression and propagation of the newly inserted TE while avoiding the most detrimental consequences for the host genome. The sites targeted by the five Ty long terminal repeat (LTR)-retrotransposons in the genome of $S$. cerevisiae are a perfect example of such a compromise. The genome of $S$. cerevisiae is particularly compact with $70 \%$ open reading frames and few intergenic spaces, which is quite exceptional for an eukaryote (Goffeau et al., 1996). In this organism, different strategies of Ty integration have evolved to avoid coding sequences and integrate into gene-poor regions. Ty5 integrates into silent chromatin at the silent mating loci $H M$ and telomeres, where they do not disrupt essential functions (Zou et al., 1996). Ty1/Ty2/Ty4 and Ty3 target RNA polymerase III (Pol III)-transcribed genes, i.e. tRNA genes ( $t D N A \mathrm{~s})$ and few additional non-coding RNA genes including 5S RNA, but interestingly do not compete for the same sequences around these genes. Ty 3 has the most specific pattern of integration within 20 base pairs upstream of the Pol III transcription start sites, while Ty1 integrates into a larger one-kilobase window upstream of these genes (Baller et al., 2012; Chalker and Sandmeyer, 1992; Devine and Boeke, 1996; Mularoni et al., 2012; Patterson et al., 2019; Qi et al., 2012). The integration preference of Ty2 and Ty4 for the same 1-kb window upstream of Pol III-transcribed genes were deduced from the distribution of their endogenous copies in the yeast genome (Carr et al., 2012; Kim et al., 1998). Because $t D N A$ s are in multicopy and thus individually nonessential, Ty1-Ty4 integration patterns minimize genetic damage to their host and yet allow these elements to replicate. More generally, TE insertions close to Pol III-transcribed genes have been described in other yeasts and organisms with compact genomes indicating that $t D N A s$ are recurrent safe landing pads for TEs (Cheung et al., 2018; Neuvéglise et al., 2002; Spaller et al., 2016). 
Transcription of Ty elements by RNA polymerase II (Pol II), mainly in haploid cells, is influenced by their location in the genome. Around 30 copies of Ty1 are present in the laboratory strain S288C. While these copies are constitutively expressed at relatively low levels in haploid cells (Curcio and Garfinkel, 1992; Morillon et al., 2002), Ty1 RNA is abundant (Curcio et al., 1990; Elder et al., 1981). Regarding Ty3, the transcription of the two endogenous copies is repressed by competing Pol III transcription of the adjacent $t D N A s$, resulting in extremely low level of expression (Bilanchone et al., 1993; Hull et al., 1994; Kinsey and Sandmeyer, 1991). S. cerevisiae does not have a functional Ty5 element but the introduction of an active Ty5 element from $S$. paradoxus revealed that the heterochromatin targeted by the element repressed the transcription of de novo Ty5 insertions (Ke et al., 1997). Yet, the expression of both Ty 3 and Ty 5 is strongly stimulated in haploid cells exposed to pheromones, allowing the transposition of active copies in particular conditions (Bilanchone et al., 1993; Ke et al., 1997; Kinsey and Sandmeyer, 1995). Switching between repression and activation of Ty expression undoubtedly may help minimize the adverse effects of their propagation while maintaining a relatively low copy number in the cells. Noteworthy, Ty1 and Ty2 are the two most abundant Ty families in S288C (Carr et al., 2012; Kim et al., 1998), suggesting that their integration in a one-kilobase window upstream of Pol III-transcribed genes may have contributed to their successful propagation in the genome. Ty4, of which there are only defective copies located in the upstream region of the $t D N A s$, has not had the same success probably because of intrinsic transcription defects (Hug and Feldmann, 1996). As Ty families are not constantly represented among the Saccharomyces genus (BleykastenGrosshans and Neuvéglise, 2011; Neuvéglise et al., 2002; Souciet et al., 2009), it would be interesting to compare the insertion patterns of successful Ty elements in other Saccharomyces species.

\section{Tethering factors dictate Ty1, Ty3 and Ty5 integration site preferences}

Ty LTR-retrotransposons replicate by reverse transcription of their RNA genome into a cDNA that is next integrated into the host genome by their own integrase (IN). Their study has highlighted a conserved evolutionary strategy to target their integration in particular regions of the genomes: the specific interaction of IN with cellular tethering factors, mainly DNA or chromatin binding proteins, present at preferred sites (for review (Kvaratskhelia et al., 2014; Sultana et al., 2017). Tethering factors have been identified for Ty1, Ty3 and Тy5 
124 (Figure 1), as well as for the Tf1 retrotransposon of Schizosaccharomyces pombe (Hickey et 125 al., 2015; Jacobs et al., 2015), the Murine Leukemia Virus (MLV) (Gupta et al., 2013; De 126 Rijck et al., 2013; Sharma et al., 2013) and the Human Immunodeficiency Virus (HIV) 127 (Cherepanov et al., 2003; Llano et al., 2006). Historically, Ty5 and Ty3 were pioneer models 128 to understand the role of tethering factors in targeted integration.

- Ty5 targets silent chromatin by interacting with Sir4. Ty5 is probably the simplest model. Precisely, Ty5 integration in heterochromatin requires an interaction between a targeting domain of six amino acids at the Ty5 integrase (IN5) C-terminus and the Sir4 heterochromatin protein (Gai and Voytas, 1998; Xie et al., 2001). When this interaction is abolished, Ty5 integrates randomly in genic regions and becomes mutagenic (Gai and Voytas, 1998). Moreover, the IN5-Sir4 interaction requires phosphorylation of a serine residue within the targeting domain, which is abolished by nutrient starvation, suggesting a control of Ty5 integration profile by stress (Dai et al., 2007). The interaction with Sir4 is sufficient to dictate Ty5 integration sites, because artificially tethering of Sir4 to ectopic DNA sites creates new Ty5 integration hot spots (Zhu et al., 2003). Conversely, swapping the Ty5 targeting domain with peptides that recognize other protein partners directs Ty5 integration to the DNA sites bound by these protein partners (Zhu et al., 2003).

\section{- Ty1 and Ty3 interact with different Pol III transcription factors to achieve their specific} targeting. For the transcription of $t D N A \mathrm{~s}$, TFIIIC recognizes the $\mathrm{A}$ and $\mathrm{B}$ boxes of their intergenic promoter, and then recruits TFIIIB to the upstream promoter region (TATA box), which finally recruits Pol III (for review (Acker et al., 2013)). Both Ty1 and Ty3 require the prior recruitment of TFIIIC for their integration but they then achieve their specific targeting by interacting with different components of the Pol III transcription system (Chalker and Sandmeyer, 1992; Devine and Boeke, 1996; Kirchner et al., 1995).

\section{Ty3 integrase interacts with Pol III general transcription factors TFIIIB and} TFIIIC. TFIIIB has been identified as the Ty3 tethering factor. Two subunits of TFIIIB, Brf1 and TBP, are sufficient for Ty3 targeted insertion in vitro while, the third one, Bdp1, only increases its efficiency (Qi and Sandmeyer, 2012; Yieh et al., 2000, 2002). Loss-ofinteraction mutants have not been characterized to definitively demonstrate the role of the interaction between TFIIIB and Ty3 integrase (IN3) in targeting the retrotransposon in vivo. However, genome-wide analyses of de novo Ty3 insertions confirmed that Ty3 integration occurs at virtually all Pol III-transcribed genes and coincides with TFIIIB bound sites (Qi et 
al., 2012). Moreover, the Tfc1 subunit of TFIIIC interacts with IN3 by two-hybrid and in vitro, and the truncation of $\mathrm{Tfc} 1 \mathrm{C}$-terminus led to Ty 3 integration in a single orientation with respect to the direction of transcription of the targeted gene (Aye et al., 2001). Yet, Ty3 does not integrate into the extra-TFIIIC genomic sites from which TFIIIB is absent, indicating that TFIIIC primary role in the targeting of Ty3 is to recruit TFIIIB (Qi et al., 2012). However, this does not preclude a contribution from TFIIIC after Ty3 recruitment. of Ty1, it took much longer to identify a factor responsible for Ty1 targeting. In 1996, the complete sequencing of a $S$. cerevisiae genome revealed that most Ty1 sequences - solo LTR and full-length elements - were located upstream of the Pol III-transcribed genes, validating earlier observations made on a limited number of Tyl endogenous copies (Eigel and Feldmann, 1982; Goffeau et al., 1996). The study of approximately 100 unselected de novo Ty1 insertions on chromosome III also suggested that this distribution resulted from an active integration preference for these regions rather than an evolutionary process occurring after Ty1 insertion (Ji et al., 1993). In the same decade, a very elegant study, based on the analysis of Ty1 integration events upstream of a reporter $t D N A$ carried on plasmid, showed that mutation of the B box of the $t D N A$ promoter prevented Tyl integration (Devine and Boeke, 1996). This observation provided a first indication that one or more components of the Pol III transcription system could be involved in Tyl integration targeting. A milestone was reached in 2012 by joint publications reporting similar approaches to set-up large-scale sequencing of de novo Ty1 insertion events. Both studies confirmed that the vast majority $(90 \%)$ of insertions occurred in a 2-kilobase window upstream of Pol III genes (Baller et al., 2012; Mularoni et al., 2012). They also validated that the Pol III machinery was required at the site of integration, since $t D N A$ genes that were poorly transcribed were not targeted. A major breakthrough followed with the description of a functional interaction between Tyl integrase (IN1) and the AC40 subunit of Pol III that we discovered by serendipity in a two-hybrid screen with AC40 as bait (Bridier-Nahmias et al., 2015). This screen also identified an interaction between AC40 and the integrases of Ty 2 and Ty 4 that display the same integration preferences as Ty1. AC40 is essential for yeast viability. Fortunately, AC40 ortholog in $S$. pombe, AC40sp, could be used as a loss-of-interaction mutant, since it complemented AC40 function in Pol III transcription but did not interact with IN1. De novo Tyl insertions were thus compared genome-wide in strains containing either AC40 or AC40sp. The replacement of AC40 by AC40sp strongly affected Ty1 integration upstream of all Pol III-transcribed 
genes, but did not alter its integration frequency. Surprisingly, in the absence of IN1-AC40 interaction, Tyl integration was not random but enriched in the subtelomeres, revealing Ty1 secondary preferred targets. Yeast subtelomeres lack essential genes but contain genes needed to respond to environmental changes (Brown et al., 2010). Targeting the integration of Ty1 in these regions could protect the yeast genome from Tyl mobility, while facilitating yeast adaptation and gene innovation in response to stress.

The key role of IN1-AC40 interaction has been further validated in a recent study, by the identification of the targeting domain of IN1 (TD1 for Targeting Domain of Tyl) (AsifLaidin et al., 2020). This domain consists of a short sequence of 5 amino acids at the Cterminus of IN1, which is conserved in Ty2 and Ty4 integrases. The position of TD1 is strikingly similar to the targeting domain of Ty5 integrase. TD1 triggers the direct interaction between IN1 and AC40 in vivo and in vitro. Disrupting the AC40-IN1 interaction by using either AC40sp or single amino acid mutants of TD1 led to the same alteration of Ty1 integration pattern, namely the loss of integration upstream of Pol III genes and the redistribution of Ty1 insertions to subtelomeres, without affecting Ty1 integration frequency. Remarkably, replacing the targeting domain of Ty5 by TD1 into the Ty5 integrase was sufficient to trigger its interaction with $\mathrm{AC} 40$ and redirect Ty5 integration from heterochromatin to the region upstream of Pol III-transcribed genes. To the best of our knowledge, it is the first evidence that the targeting domain of a retroelement is functional to re-target another retroelement to specific sites. This could set the basis for engineering chimeric retroviral integrases that could integrate transgenes into safe and well-characterized genomic sites.

- Is AC40 the only Pol III subunit involved in Ty1 integration targeting? AC40 is common to Pol III and Pol I. In our recent study, we set up ChIP-seq conditions to survey all IN1

213 binding sites in the genome and showed that IN1 is present at all the sites naturally occupied 214 by both polymerases (Asif-Laidin et al., 2020). IN1 was not detected at other regions of the genome, suggesting that IN1 binding to chromosomes is constrained to the regions where AC40 is present. A similar profile was observed for Ty5 integrase carrying Ty1 TD1, further

217 demonstrating that binding to AC40 is sufficient for the recruitment to Pol I and Pol III 218 occupied loci. However, the fact that Ty1 integrates only at Pol III-transcribed genes and not 219 at Pol I-transcribed genes suggests that additional factors either prevent Ty1 integration at Pol 220 I-transcribed genes or help it at Pol III-transcribed genes. Pol III is composed of 17 subunits. 221 Ten have counterparts in the three RNA polymerases, two are shared with Pol I (AC40 and 
AC19) and have counterparts in Pol II (Rpb3 and Rpb11), and five are specific to Pol III and organized in two sub-complexes $\mathrm{C} 82 / \mathrm{C} 31 / \mathrm{C} 34$ and $\mathrm{C} 37 / \mathrm{C} 53$ equivalent to TFIIE and TFIIF in Pol II transcription (for review (Vannini and Cramer, 2012)). A recent proteomic screen to identify IN1 co-factors by mass spectrometry has recovered AC40 and four Pol III subunits (C25, C31, C34, C53) (Cheung et al., 2016). Since Pol III is a stable complex, immunoprecipitation of a particular subunit results in the co-immunoprecipitation of the whole complex (Oficjalska-Pham et al., 2006), making it difficult to ascertain whether IN1 interacts directly with different Pol III subunits. A weak interaction was also detected in vitro between IN1 and Pol III specific subunits C31, C34 and C53 (Cheung et al., 2016). The structure of the IN/cDNA pre-integration complex, referred to as the intasome, has been characterized for several retroviruses in the past years. These complexes contain a multiple of 4 INs, with structurally and functionally separate roles, combined to a single cDNA molecule (for review (Lesbats et al., 2016)). Although the structure of the Ty1 intasome has not yet been described, the assembly of at least four integrases on the Ty1 cDNA could explain the functionally distinct interactions of IN1 with different Pol III subunits. However, we showed that the co-immunoprecipitation of IN1 with Pol III in vivo was dependent on the IN1-AC40 interaction and that IN1 association with $\mathrm{C} 34$ and C53 could not be detected outside the Pol III complex (Asif-Laidin et al., 2020). This indicates that the interaction with C34 and C53 and probably C31 requires IN1 to be recruited by AC40 to Pol III first. Cheung et al. showed that thermosensitive mutants of C31, C34 and C53 decreased the integration of endogenous Ty1 elements upstream of the SUF16 reporter gene (Cheung et al., 2016). The C31 and C34 mutants also slightly decreased the overall frequency of Ty1 integration, which is not the case for AC40-IN1 loss-of-interaction mutants, and the C53 mutants affected $t D N A$ transcription efficiency. Thus, it cannot be excluded that these mutants alter the structure of Pol III and consequently the IN1-AC40 interaction or other steps of Ty1 integration that would depend on a fully functional Pol III.

A cryosensitive mutant of $\mathrm{C} 53\left(\mathrm{C} 53^{\Delta 2-280}\right.$, slow growth at $\left.20^{\circ} \mathrm{C}\right)$ has been studied in more detail to explore the role of this subunit in Ty1 integration (Cheung et al., 2016). This mutant has an unexpected impact on Ty1 integration preferences. While the C53 ${ }^{\Delta 2-280}$ mutant led to a complete loss of integration of Ty1 elements upstream of an endogenous $t D N A$, it did not induce the redistribution of Ty1 integration events to subtelomeres, as observed in AC40-IN loss-of interaction mutants. However, C53 $3^{\Delta 2-280}$ mutant only marginally alters Ty1 integration frequency ( $80 \%$ from WT frequency). These different effects on Ty1 are difficult to reconcile. 
255 High-throughput sequencing data of de novo Tyl insertion sites would allow determining 256 where Tyl integrates in this mutant. It is therefore not clear at this time if and how, beside AC40, other Pol III subunits are involved in Ty1 integration targeting in vivo. Further studies, in particular structural analyses, will be necessary to address the role of the entire Pol III complex, and especially of C31, C34 and C53, in Ty1 integration.

- How does the interaction of Ty1 and Ty3 integrases with their respective tethering factors explain why Ty1 and Ty3 target non-overlapping genomic regions?

Most Ty3 insertions occur within 20 base pairs upstream of $t D N A$ s. The tethering factor of Ty3, TFIIIB, binds $t D N A$ promoters between positions -40 to -5 relative to the transcription start site (TSS) in a very stable manner and possibly remains bound throughout the transcription cycle (Leśniewska and Boguta, 2017)). This characteristic would allow Ty3 to integrate at any stage of the Pol III transcription. However, Pol III could interfere with the TFIIIB/IN3 interaction and Ty3 integration process, since Pol III and Ty3 binding to the TFIIIB complex share similarities and compete in vitro (Connolly and Sandmeyer, 1997; Yieh et al., 2002). The integration of Ty3 could thus be promoted by the structural changes induced at the elongation step, when TFIIIB remains bound to the promoter and Pol III moves on the gene, even though the two complexes remain associated.

272 Unlike Ty3, the integration of Ty1 requires the presence of Pol III and takes place upstream of the region occupied by TFIIIB. AC40 is located on the surface of the Pol III complex, relatively close to the DNA that is upstream of TFIIIB binding, where Ty1 integrates. The C31, C34, and C53 subunits, which interact in vitro with IN1, are also well positioned in the Pol III structure to participate in the specificity of Ty1 integration upstream of Pol IIItranscribed genes. DNA-protein crosslinking studies and cryo-EM structures indicate that C34 contacts the promoter DNA and interacts with the Bdp1 subunit of TFIIIB (Abascal-Palacios et al., 2018; Brun et al., 1997; Han et al., 2018; Vorländer et al., 2018). In addition, the 270 amino-terminal residues of $\mathrm{C} 53$, which seem to be necessary for in vivo targeting of Ty1 integration (Cheung et al., 2016), are involved in interactions with different Pol III subunits including C82 and C34, which could bring C53 close to the upstream DNA (Wu et al., 2011).

283 Structural data of the IN1/Pol III interaction would undoubtedly lead to significant advances 284 in the understanding of the interaction of IN1 with the entire Pol III complex and the Ty1 285 integration upstream of Pol III-transcribed genes. 

selection

\section{- The role of the chromatin structure at the site of integration is important but still poorly} understood. Ty retrotransposons have preferences for particular chromatin configurations at the sites where they are recruited by their tethering factor. Ty5 preferentially integrates into nucleosome-free heterochromatin and euchromatin regions (Baller et al., 2011). The Pol III transcription start sites, which are targeted by Ty3, are also devoid of nucleosomes (Kumar and Bhargava, 2013). In contrast, Ty1 integrates into nucleosomes, stably positioned by the Isw2 chromatin-remodeling factor upstream of the genes transcribed by Pol III (Bachman et al., 2005; Gelbart et al., 2005). The preference of Ty3 and Ty1 for nucleosome-depleted and nucleosome-enriched regions, respectively, could explain in part their different profiles of integration at Pol III-transcribed genes. The Tyl integration profile upstream of $t D N A s$ shows a periodicity of $70 \mathrm{bp}$ between each insertion hotspot, corresponding to two insertion sites per nucleosome (Bachman et al., 2004). Comparison of these insertion sites with the position of the DNA in a nucleosome indicates that both sites are located on the same side of the nucleosome near the H2A-H2B interface (Baller et al., 2012; Mularoni et al., 2012). Ty1 preference for this nucleosomal position might be due to the greater extrusion of DNA or/and a lower energy barrier at this location, as suggested by structural studies of nucleosomebound intasome of the Prototype Foamy Virus (PFV) (Maskell et al., 2015; Michieletto et al., 2019; Wilson et al., 2019). Furthermore, Ty1 integration sites are slightly offset from the dyad axis of symmetry of the nucleosome (Baller et al., 2012; Mularoni et al., 2012). This asymmetry could be due to the intervention of an as yet unidentified chromatin-remodelling factor in Tyl integration or could be the consequence of the extrusion and sliding of the nucleosomal DNA induced by the integration reaction (Wilson et al., 2019).

The interaction with tethering factors does not fully explain Ty1, Ty3 and Ty5 integration profiles at the genome-wide level. For instance, nucleosome-free regions targeted by Ty 5 do not systematically correspond to Sir4 binding sites (Baller et al., 2011). Similarly, AC40 and TFIIIB are present at most $t D N A$ loci and yet Ty1 and Ty 3 do not target all $t D N A$ s with the same efficiency (Bachman et al., 2004; Mularoni et al., 2012; Patterson et al., 2019). Comparison of hot and cold $t D N A$ s for Ty 3 targeting suggests that the curvature of the DNA upstream of the TSS could interfere with the integration (Patterson et al., 2019). The dispersion of Ty1 insertion sites in the subtelomeres, when the interaction with AC40 is suppressed, is also suggestive of a special nucleosome configuration or a histone mark 
specifically enriched at this location that would help Ty1 integration (Asif-Laidin et al., 2020;

320 Bridier-Nahmias et al., 2015). In these genomic regions, Ty1 insertion profile overlaps with the extended silent chromatin domains defined by overexpression of the Sir3 protein and characterized by a high occupancy of phosphorylated H2A $(\gamma \mathrm{H} 2 \mathrm{~A})$ and a low occupancy of K79 trimethylated histone $\mathrm{H} 3$ (H3K79me3) (Hocher et al., 2018). $ү \mathrm{H} 2 \mathrm{~A}$ is also present at $t D N A \mathrm{~s}$, which are Ty1 preferred targets (Szilard et al., 2011). Several studies suggest that $\mathrm{\gamma H}_{2} \mathrm{~A}$ reduces nucleosome stability and makes the DNA double helix more accessible to other factors (Downs et al., 2000; Sharma et al., 2019). Nucleosomes containing $\mathrm{\gamma H} 2 \mathrm{~A}$ could therefore facilitate Ty1 integration. On the other hand, genome-wide analysis of Tyl de novo insertions also points to a correlation with sites enriched in H2A.Z, a signature also observed for several Ty1/copia like element in plants (Mularoni et al., 2012; Quadrana et al., 2019). However, H2A.Z relative occupancy could not explain Tyl cold and hot Pol-III transcribed targets (Mularoni et al., 2012) and if H2A.Z was identified as necessary for Ty1 transposition in a genetic screen (Dakshinamurthy et al., 2010), we were unable to reproduce the observation in independent $h t z 1 \Delta$ reconstructed strains (unpublished data). At this time, we cannot conclude whether the presence of these histone marks at Tyl insertion sites is causative or simply correlative. Several genes with functions related to chromatin modification or remodeling have been identified to regulate Ty1 retrotransposition efficiency in genetic screens (reviewed in (Curcio et al., 2015)). Among these genes, the role in Ty1 integration targeting was studied by genome-wide approaches for those encoding the Rtt109 histone acetyl-transferase (H3K79 and H3K9), the Hos2 histone H3 and H4 deacetylases or the Rad6 ubiquitin-conjugating enzyme (Baller et al., 2012). Only rad6 4 had a significant alteration in the integration profile with more Ty1 insertions in ORFs, without altering overall Ty1 integration at Pol III-transcribed genes. However, differences in Ty1 integration between $t D N A s$ were not specifically investigated for all these mutants, as was done with Ty3 by

344 Patterson et al. (Patterson et al., 2019). More resolutive analysis will be required to determine whether Rad6, along with other factors, contribute to the variation in Ty1 integration between

346 Pol III-transcribed genes. Deciphering the molecular bases of Ty1 integration at subtelomeres 347 would undoubtedly help to address the role of chromatin in Ty1 integration site selection.

348 Other aspects of the chromosome structure could also influence Ty1 integration. Indeed, Pol 349 III-transcribed genes, as sites of strong transcriptional activity, have been described as cohesin 350 binding sites (D’Ambrosio et al., 2008; Lengronne et al., 2004; Ocampo-Hafalla and 351 Uhlmann, 2011). Cohesins are involved in chromosome compaction, mediate chromosome 
352 loop formation and hold sister chromatids during the G2 and M phases of the cell cycle 353 (Costantino et al., 2020; Gligoris et al., 2014; Haering et al., 2008). Interestingly, the protease 354 separase Esp1, which cleaves the cohesin complex at the anaphase onset allowing the sister chromatids to segregate into separate daughter cells, interacts physically with IN1 (Ho et al., 2015). Cohesin or Esp1 mutants that affect cohesin removal, decrease the frequency of Ty1 integration and its targeting upstream of the SUF16 tRNA gene (Ho et al., 2015). This observation suggests that the integration of Tyl could be favored during the $\mathrm{M}$ phase, when cohesins are absent from $t D N A \mathrm{~s}$ and would be consistent with the difference in retrotransposition frequency between mother and daughter cells (Patterson et al., 2015).

- Could the nuclear import of Ty1 IN:cDNA complexes and the integration process be coupled to promote integration at Pol III-transcribed genes? To complete their replication cycle, retroelements need to traffic in and out of the nucleus. The nuclear envelope is therefore a physical barrier to integrate a new copy into the host genome for all retroelements. In particular, for lentiviruses, such as HIV-1, that infect non-dividing cells as well as for retrotransposons in yeasts, whose nuclear envelope does not break down during mitosis, Nuclear Pore Complexes (NPCs) are essential gatekeeper to control access to genomic DNA. NPCs are large macromolecular complexes embedded within the nuclear envelope and built of multiple copies of about 30 different proteins, called nucleoporins (Nups), that are organized into modular subcomplexes (reviewed in (Lin and Hoelz, 2019)). Not surprisingly, many links have long been noticed between the replication cycle of retroelements and NPCs (Figure 2A). Indeed, the NPC has been early described as the main route for the nuclear export of viral mRNAs and the nuclear import of pre-integration complexes (PICs) (reviewed in (Molenberghs et al., 2020)). The role played by the NPCs and other cellular factors in nuclear import of HIV-1 PIC has been particularly well studied (reviewed in (Bhargava et al., 2018)). On the contrary, only a few studies have investigated the nuclear import process of Ty retrotransposons. The nuclear localization signal driving PIC nuclear import has been identified in the C-terminal region of both Ty1 and Ty3 integrases (Kenna et al., 1998; Lin et al., 2001; Moore et al., 1998). In both cases, NLS have been characterized as bipartite NLS (bNLS) and IN1 has been demonstrated to be imported by the classical nuclear protein import pathway (McLane et al., 2008). Yet, the bipartite NLS of IN1 is atypical with a particularly long linker of 29 amino acids separating the two clusters of basic amino acids, as compared to the usual 9-12 amino acid long linkers. It has been shown that for such a long linker to be functional, its sequence and flexibility properties are of particular importance (Lange et al., 
2010). Interestingly, the targeting domain of Ty1 (TD1) is located within this unconventional linker (Asif-Laidin et al., 2020). We have shown that Ty1 nuclear import and integration targeting are two distinct functions but it is tempting to speculate on the evolutionary advantages that the combination of sequences required for PIC nuclear import and integration targeting could represent (Figure 2B). The binding of the two basic amino acid-rich regions of IN1 bNLS to the two NLS-binding pockets of importin- $\alpha$ (Kosugi et al., 2009; Lange et al., 2010) could perhaps expose the TD1 to AC40. Once in the nucleus, the interaction with AC40 would help Ran-GTP to dissociate the IN1-importin- $\alpha / \beta$ complex (Rothenbusch et al., 2012). This coupling between nuclear entry and Pol III interaction could promote Ty1 targeting to $t D N A \mathrm{~s}$.

In addition, evidence has accumulated that NPCs are key platforms in the 3D organization of the genome (reviewed in (Canat et al., 2020)). In yeast, an association of $t D N A s$ with NPCs mediated by the nuclear basket nucleoporins has been reported during the peak of $t D N A$ transcription (i.e. M phase) (Chen and Gartenberg, 2014). A current model therefore suggests a direct role of Nups in coupling nuclear entry and integration of retroelements into genomic loci in the close proximity of NPCs. This model was mainly based on the study of HIV-1 integration preferences and their modification when the nuclear basket of the NPC was altered (Lelek et al., 2015; Lusic and Siliciano, 2017; Marini et al., 2015). Several Nups have been identified in large-scale screens as regulating Ty1 and Ty3 retrotransposition rate (Curcio et al., 2015; Irwin et al., 2005). However, the association of $t D N A s$ with NPCs suggests that, as with HIV-1, NPCs could also directly influence Ty integration preferences. Consistent with this, the alteration of the nuclear pore basket was recently shown to reduce Ty1 integration upstream of two distinct tDNAs (Manhas et al., 2018). This reduction in nuclear basket

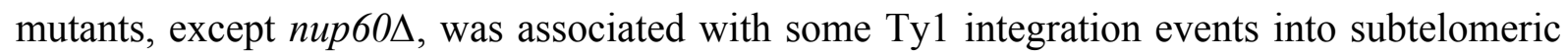
loci, although less than in the case of IN1-AC40 loss of interaction. However, recent studies on the important role played by CPSF6 in HIV-1 integration patterns has questioned this model of coupling between PIC nuclear import and integration into genomic targets in the direct vicinity of the NPCs (Achuthan et al., 2018; Bejarano et al., 2019; Buffone et al., 2018). Similarly, genome-wide analysis of a high number of Tyl de novo integration events will be necessary to finally uncover the role played by the nuclear pore basket in Ty1 integration upstream of $t D N A s$. 
418 As demonstrated by the study of the Ty retrotransposons, tethering factors undoubtedly play a

419 fundamental role in the integration site selection of retroelements. However, it is quite 420 obvious that other actors come into play to define locally the most accessible sites among the 421 preferred targets. How Ty integrases interact with their tethering factors and how these 422 interactions influence Ty integration at the target site has not been explored at the structural 423 levels, yet. However, this would be informative, especially in the case of Ty1 to decipher the 424 roles played by the different subunits of Pol III interacting with IN1 and to challenge the 425 coupling model between nuclear import and interaction with AC40. Ty1 differs from Ty3 and 426 Ty5, as it targets nucleosomal DNA. High-throughput sequencing of Ty1 de novo insertion events combined with high-resolution mapping of DNA binding sites for histone marks and chromatin factors will certainly give important insights into the chromatin factors participating to Ty1 targeting. Genome-wide approaches in different mutants affecting the NPC or cohesin functions will also help to understand their respective role in Ty1 integration targeting. Finally, the knowledge acquired in recent years with Ty retrotransposons raises new challenging questions about the ballet of players involved in integration targeting and definitely demonstrates how retrotransposons in yeasts remain relevant and powerful models to explore at the molecular levels the intimate relationship between TEs and their host.

\section{Acknowledgments}

437 We apologize to the many scientists that we may have omitted to mention in this review due 438 due to space limitations. The authors thank J. Acker, A. Asif-Laidin, J. Curcio and E. Fabre 439 for the critical reading of the manuscript.

\section{Declarations}

442 Funding. This work was supported by intramural funding from Centre National de la 443 Recherche Scientifique (CNRS), the Université of Paris and the Institut National de la Santé 444 et de la Recherche Médicale (INSERM), and from grants from the Fondation ARC pour la 445 Recherche sur le Cancer (PJA 20151203412 and PJA 20191209703), the Agence Nationale 446 de la Recherche through the generic call project ANR-17-CE11-0025. AB was supported by a 447 post-doctoral fellowship from the ANR through the initiatives d'excellence (Idex ANR-11- 
448 IDEX-0005-02) and the Labex "Who am I?" (ANR11-LABX-0071).

449 Conflict of interests/Competing interests. Not applicable.

450 Availability of data and material. Not applicable.

451 Code availability. Not applicable.

452 Authors' contributions. Equal contribution.

453 


\section{References}

455 Abascal-Palacios, G., Ramsay, E.P., Beuron, F., Morris, E., and Vannini, A. (2018).

456 Structural basis of RNA polymerase III transcription initiation. Nature 553, 301-306.

457 Achuthan, V., Perreira, J.M., Sowd, G.A., Puray-Chavez, M., McDougall, W.M., Paulucci-

458 Holthauzen, A., Wu, X., Fadel, H.J., Poeschla, E.M., Multani, A.S., et al. (2018). Capsid-

459 CPSF6 Interaction Licenses Nuclear HIV-1 Trafficking to Sites of Viral DNA Integration.

460 Cell Host Microbe 24, 392-404.e8.

461 Acker, J., Conesa, C., and Lefebvre, O. (2013). Yeast RNA polymerase III transcription

462 factors and effectors. Biochim. Biophys. Acta - Gene Regul. Mech. 1829, 283-295.

463 Asif-Laidin, A., Conesa, C., Bonnet, A., Grison, C., Adhya, I., Menouni, R., Fayol, H.,

464 Palmic, N., Acker, J., and Lesage, P. (2020). A small targeting domain in Ty1 integrase is

465 sufficient to direct retrotransposon integration upstream of tRNA genes. EMBO J. 39, 1-17.

466 Aye, M., Dildine, S.L., Claypool, J.A., Jourdain, S., and Sandmeyer, S.B. (2001). A

467 truncation mutant of the 95-kilodalton subunit of transcription factor IIIC reveals asymmetry 468 in Ty3 integration. Mol Cell Biol 21, 7839-7851.

469 Aye, M., Irwin, B., Beliakova-Bethell, N., Chen, E., Garrus, J., and Sandmeyer, S. (2004).

470 Host factors that affect Ty3 retrotransposition in Saccharomyces cerevisiae. Genetics 168, $471 \quad 1159-1176$.

472 Bachman, N., Eby, Y., and Boeke, J.D. (2004). Local definition of Ty1 target preference by 473 long terminal repeats and clustered tRNA genes. Genome Res 14, 1232-1247.

474 Bachman, N., Gelbart, M.E., Tsukiyama, T., and Boeke, J.D. (2005). TFIIIB subunit Bdp1p is 475 required for periodic integration of the Ty1 retrotransposon and targeting of Isw $2 p$ to $S$.

476 cerevisiae tDNAs. Genes Dev 19, 955-964.

477 Baller, J.A., Gao, J., and Voytas, D.F. (2011). Access to DNA establishes a secondary target 478 site bias for the yeast retrotransposon Ty5. Proc Natl Acad Sci U S A 108, 20351-20356.

479 Baller, J.A., Gao, J., Stamenova, R., Curcio, M.J., and Voytas, D.F. (2012). A nucleosomal 480 surface defines an integration hotspot for the Saccharomyces cerevisiae Ty1 retrotransposon. 481 Genome Res 22, 704-713. 
482

483

484

485

486

487

488

489

490

491

492

493

494

495

496

497

498

499

500

501

502

503

504

505

506

507

508

Bejarano, D.A., Peng, K., Laketa, V., Bo, K., Lusic, M., and Mu, B. (2019). HIV-1 nuclear import in macrophages is regulated by CPSF6-capsid interactions at the nuclear pore complex. Elife 8, e41800.

Bhargava, A., Lahaye, X., and Manel, N. (2018). Let me in: Control of HIV nuclear entry at the nuclear envelope. Cytokine Growth Factor Rev. 40, 59-67.

Bilanchone, V.W., Claypool, J.A., Kinsey, P.T., and Sandmeyer, S.B. (1993). Positive and negative regulatory elements control expression of the yeast retrotransposon Ty3. Genetics $134,685-700$.

Bleykasten-Grosshans, C., and Neuvéglise, C. (2011). Transposable elements in yeasts.

Comptes Rendus - Biol. 334, 679-686.

Bridier-Nahmias, A., Tchalikian-Cosson, A., Baller, J.A., Menouni, R., Fayol, H., Flores, A., Saïb, A., Werner, M., Voytas, D., and Lesage, P. (2015). An RNA polymerase III subunit determines sites of retrotransposon integration. Science. 348, 585-588.

Britten, R.J., and Davidson, E.H. (1969). Gene regulation for higher cells: A theory. Science. $165,349-357$.

Brown, C. a., Murray, A.W., and Verstrepen, K.J. (2010). Rapid expansion and functional divergence of subtelomeric gene families in yeasts. Curr. Biol. 20, 895-903.

Brun, I., Sentenac, A., and Werner, M. (1997). Dual role of the C34 subunit of RNA polymerase III in transcription initiation. EMBO J. 16, 5730-5741.

Buffone, C., Martinez-Lopez, A., Fricke, T., Opp, S., Severgnini, M., Cifola, I., Frabetti, S., Skorupka, K., Zadrozny, K.K., Ganser-Pornillos, B.K., et al. (2018). Nup153 Unlocks the Nuclear Pore Complex for HIV-1 Nuclear Import in Non-Dividing Cells. J. Virol. 92, 1-29.

Burns, K.H. (2017). Transposable elements in cancer. Nat. Rev. Cancer 17, 415-424.

Bushman, F.D. (2003). Targeting survival: Integration site selection by retroviruses and LTRRetrotransposons. Cell 115, 135-138.

Canat, A., Veillet, A., Bonnet, A., and Therizols, P. (2020). Genome anchoring to nuclear landmarks drives functional compartmentalization of the nuclear space. Br. Funct Genomics 
$19,101-110$.

510 Carr, M., Bensasson, D., and Bergman, C.M. (2012). Evolutionary Genomics of Transposable 511 Elements in Saccharomyces cerevisiae. PLoS One 7, e50978.

512 Chalker, D.L., and Sandmeyer, S.B. (1992). Ty3 integrates within the region of RNA 513 polymerase III transcription initiation. Genes Dev 6, 117-128.

514 Chen, M., and Gartenberg, M.R. (2014). Coordination of tRNA transcription with export at 515 nuclear pore complexes in budding yeast. Genes Dev. 28, 959-970.

516 Cherepanov, P., Maertens, G., Proost, P., Devreese, B., Van Beeumen, J., Engelborghs, Y., 517 De Clercq, E., and Debyser, Z. (2003). HIV-1 integrase forms stable tetramers and associates 518 with LEDGF/p75 protein in human cells. J. Biol. Chem. 278, 372-381.

519 Cheung, S., Ma, L., Chan, P.H.W., Hu, H.-L., Mayor, T., Chen, H.-T., and Measday, V. 520 (2016). Ty1-Integrase interacts with RNA Polymerase III specific subcomplexes to promote 521 insertion of Ty1 elements upstream of Pol III-transcribed genes. J. Biol. Chem. 291, 63965226411.

523 Cheung, S., Manhas, S., and Measday, V. (2018). Retrotransposon targeting to RNA 524 polymerase III-transcribed genes. Mob. DNA 9, 1-15.

525 Chuong, E.B., Elde, N.C., and Feschotte, C. (2016). Regulatory activities of transposable 526 elements: from conflicts to benefits. Nat Rev Genet 18, 71-86.

527 Connolly, C.M., and Sandmeyer, S.B. (1997). RNA-Polymerase-III Interferes with Ty3 528 Integration. FEBS Lett 405, 305-311.

529 Cosby, R.L., Chang, N., and Feschotte, C. (2019). Host - transposon interactions : conflict , 530 cooperation, and cooption. Genes Dev. 33, 1098-1116.

531 Costantino, L., Hsieh, T.-H., Lamothe, R., Darzacq, X., and Koshland, D. (2020). Cohesin 532 residency determines chromatin loop patterns. Elife 9, e59889.

533 Curcio, M.J., and Garfinkel, D.J. (1992). Posttranslational control of Ty1 retrotransposition 534 occurs at the level of protein Posttranslational Control of Tyl Retrotransposition Occurs at the 535 Level of Protein Processing. Molec. Cell. Biol. 12, 2813-2825. 
536 Curcio, M.J., Hedge, A.M., Boeke, J.D., and Garfinkel, D.J. (1990). Ty RNA levels determine

537 the spectrum of retrotransposition events that activate gene expression in Saccharomyces 538 cerevisiae. Mol. Gen. Genet. 220, 213-221.

539 Curcio, M.J., Lesage, P., and Lutz, S. (2015). The Ty1 LTR-Retrotransposon of Budding 540 Yeast, Saccharomyces cerevisiae. Mob. DNA III 927-964.

541 D’Ambrosio, C., Schmidt, C.K., Katou, Y., Kelly, G., Itoh, T., Shirahige, K., and Uhlmann, 542 F. (2008). Identification of cis-acting sites for condensin loading onto budding yeast 543 chromosomes. Genes Dev. 22, 2215-2227.

544 Dai, J., Xie, W., Brady, T.L., Gao, J., and Voytas, D.F. (2007). Phosphorylation regulates 545 integration of the yeast Ty5 retrotransposon into heterochromatin. Mol Cell 27, 289-299.

546 Dakshinamurthy, A., Nyswaner, K.M., Farabaugh, P.J., and Garfinkel, D.J. (2010). BUD22 547 affects Ty1 retrotransposition and ribosome biogenesis in Saccharomyces cerevisiae. Genetics $548 \quad 185,1193-1205$.

549 Devine, S.E., and Boeke, J.D. (1996). Integration of the yeast retrotransposon Ty1 is targeted 550 to regions upstream of genes transcribed by RNA polymerase III. Genes Dev. 10, 620-633.

551 Downs, J. a, Lowndes, N.F., and Jackson, S.P. (2000). A role for Saccharomyces cerevisiae 552 histone H2A in DNA repair. Nature 408, 1001-1004.

553 Eigel, A., and Feldmann, H. (1982). Ty1 and delta elements occur adjacent to several tRNA 554 genes in yeast. EMBO J. 1, 1245-1250.

555 Elder, R.T., St. John, T.P., Stinchcomb, D.T., Davis, R.W., and Scherer, S. (1981). Studies on 556 the transposable element Ty1 of yeast. Cold Spring Harb. Symp. Quant. Biol. 581-591.

557 Gai, X., and Voytas, D.F. (1998). A single amino acid change in the yeast retrotransposon 558 Ty5 abolishes targeting to silent chromatin. Mol Cell 1, 1051-1055.

559 Gelbart, M.E., Bachman, N., Delrow, J., Boeke, J.D., and Tsukiyama, T. (2005). Genome560 wide identification of Isw 2 chromatin-remodeling targets by localization of a catalytically 561 inactive mutant. Genes Dev. 19, 942-954.

562 Gligoris, T.G., Scheinost, J.C., Bürmann, F., Petela, N., Chan, K.L., Uluocak, P., Beckouët, 
563 F., Gruber, S., Nasmyth, K., and Löwe, J. (2014). Closing the cohesin ring: Structure and 564 function of its Smc3-kleisin interface. Science. 346, 963-967.

565 Goffeau, A., Barrell, B.G., Bussey, H., Davis, R.W., Dujon, B., Feldmann, H., Galibert, F., 566 Hoheisel, J.D., Jacq, C., Johnston, M., et al. (1996). Life with 6000 genes. Science (80-. ). $567274,546-567$.

568 Griffith, J.L., Coleman, L.E., Raymond, A.S., Goodson, S.G., Pittard, W.S., Tsui, C., and 569 Devine, S.E. (2003). Functional Genomics Reveals Relationships Between the Retrovirus570 Like Ty1 Element and Its Host Saccharomyces cerevisiae. Genetics 164, 867-879.

571 Gupta, S.S., Maetzig, T., Maertens, G.N., Sharif, A., Rothe, M., Weidner-Glunde, M., Galla, 572 M., Schambach, A., Cherepanov, P., and Schulz, T.F. (2013). Bromo- and extraterminal 573 domain chromatin regulators serve as cofactors for murine leukemia virus integration. J. 574 Virol. 87, 12721-12736.

575 Haering, C.H., Farcas, A.M., Arumugam, P., Metson, J., and Nasmyth, K. (2008). The 576 cohesin ring concatenates sister DNA molecules. Nature 454, 297-301.

577 Han, Y., Yan, C., Fishbain, S., Ivanov, I., and He, Y. (2018). Structural visualization of RNA 578 polymerase III transcription machineries. Cell Discov. 4, 1-15.

579 Hancks, D.C., and Kazazian, H.H. (2012). Active human retrotransposons: variation and 580 disease. Curr. Opin. Genet. Dev. 22, 191-203.

581 Hickey, a., Esnault, C., Majumdar, A., Chatterjee, a. G., Iben, J.R., McQueen, P.G., Yang, 582 a. X., Mizuguchi, T., Grewal, S.I.S., and Levin, H.L. (2015). Single Nucleotide Specific 583 Targeting of the Tf1 Retrotransposon Promoted by the DNA-Binding Protein Sap1 of 584 Schizosaccharomyces pombe. Genetics 201, 905-924.

585 Ho, K.L., Ma, L., Cheung, S., Manhas, S., Fang, N., Wang, K., Young, B., Loewen, C., 586 Mayor, T., and Measday, V. (2015). A Role for the Budding Yeast Separase, Esp1, in Ty1 587 Element Retrotransposition. PLoS Genet. 11, e1005109.

588 Hocher, A., Ruault, M., Kaferle, P., Descrimes, M., Garnier, M., Morillon, A., and Taddei, A. 589 (2018). Expanding heterochromatin reveals discrete subtelomeric domains delimited by 590 chromatin landscape transitions. Genome Res. 28, 1867-1881. 
591 Hug, A.M., and Feldmann, H. (1996). Yeast Retrotransposon Ty4 - The Majority of the Rare

592 Transcripts Lack a U3-R Sequence. Nucleic Acids Res 24, 2338-2346.

593 Hull, M.W., Erickson, J., Johnston, M., and Engelke, D.R. (1994). tRNA genes as

594 transcriptional repressor elements. Mol. Cell. Biol. 14, 1266-1277.

595 Irwin, B., Aye, M., Baldi, P., Beliakova-bethell, N., Cheng, H., Dou, Y., Liou, W., and 596 Sandmeyer, S. (2005). Retroviruses and yeast retrotransposons use overlapping sets of host 597 genes. Genome Res 15, 641-654.

598 Jacobs, J.Z., Rosado-Lugo, J.D., Cranz-Mileva, S., Ciccaglione, K.M., Tournier, V., and 599 Zaratiegui, M. (2015). Arrested replication forks guide retrotransposon integration. Science. $600349,1549-1553$.

601 Ji, H., Moore, D.P., Blomberg, M.A., Braiterman, L.T., Voytas, D.F., Natsoulis, G., and 602 Boeke, J.D. (1993). Hotspots for unselected Ty1 transposition events on yeast chromosome 603 III are near tRNA genes and LTR sequences. Cell 73, 1007-1018.

604 Ke, N., Irwin, P.A., and Voytas, D.F. (1997). The pheromone response pathway activates 605 transcription of Ty5 retrotransposons located within silent chromatin of Saccharomyces 606 cerevisiae. EMBO J 16, 6272-6280.

607 Kenna, M.A., Brachmann, C.B., Devine, S.E., and Boeke, J.D. (1998). Invading the Yeast 608 Nucleus: a Nuclear Localization Signal at the C Terminus of Ty1 Integrase Is Required for 609 Transposition In Vivo. Mol. Cell. Biol. 18, 1115-1124.

610 Kim, J.M., Vanguri, S., Boeke, J.D., Gabriel, A., and Voytas, D.F. (1998). Transposable 611 elements and genome organization: A comprehensive survey of retrotransposons revealed by 612 the complete Saccharomyces cerevisiae genome sequence. Genome Res. 8, 464-478.

613 Kinsey, P.T., and Sandmeyer, S.B. (1991). Adjacent pol II and pol III promoters: transcription 614 of the yeast retrotransposon Ty3 and a target tRNA gene. Nucleic Acids Res 19, 1317-1324.

615 Kinsey, P.T., and Sandmeyer, S.B. (1995). Ty3 transposes in mating populations of yeast: a 616 novel transposition assay for Ty3. Genetics 139, 81-94.

617 Kirchner, J., Connolly, C.M., and Sandmeyer, S.B. (1995). Requirement of RNA polymerase 618 III transcription factors for in vitro position-specific integration of a retroviruslike element. 
620 Kosugi, S., Hasebe, M., Matsumura, N., Takashima, H., Miyamoto-Sato, E., Tomita, M., and 621 Yanagawa, H. (2009). Six classes of nuclear localization signals specific to different binding grooves of importin $\alpha$. J. Biol. Chem. 284, 478-485.

623

624 625

626

Kumar, Y., and Bhargava, P. (2013). A unique nucleosome arrangement, maintained actively by chromatin remodelers facilitates transcription of yeast tRNA genes. BMC Genomics 14, 402.

Kvaratskhelia, M., Sharma, A., Larue, R.C., Serrao, E., and Engelman, A. (2014). Molecular mechanisms of retroviral integration site selection. Nucleic Acids Res 42, 10209-10225.

Lange, A., McLane, L.M., Mills, R.E., Devine, S.E., and Corbett, A.H. (2010). Expanding the definition of the classical bipartite nuclear localization signal. Traffic 11, 311-323.

Lelek, M., Casartelli, N., Pellin, D., Rizzi, E., Souque, P., Severgnini, M., Di Serio, C., Fricke, T., Diaz-Griffero, F., Zimmer, C., et al. (2015). Chromatin organization at the nuclear pore favours HIV replication. Nat. Commun. 6, 6483-6494.

Lengronne, A., Katou, Y., Mori, S., Yokabayashi, S., Kelly, G.P., Ito, T., Watanabe, Y., Shirahige, K., and Uhlmann, F. (2004). Cohesin relocation from sites of chromosomal loading to places of convergent transcription. Nature 430, 573-578.

Lesbats, P., Engelman, A.N., and Cherepanov, P. (2016). Retroviral DNA Integration. Chem. Rev. 116, 12730-12757.

Leśniewska, E., and Boguta, M. (2017). Novel layers of RNA polymerase III control affecting tRNA gene transcription in eukaryotes. Open Biol. Feb;7(2):170001. doi: $10.1098 /$ rsob.170001.

Levin, H.L., and Moran, J. V (2011). Dynamic interactions between transposable elements and their hosts. Nat Rev Genet 12, 615-627.

Lin, D.H., and Hoelz, A. (2019). The structure of the nuclear pore complex (An Update). Annu. Rev. Biochem. 88, 725-783.

Lin, S.S., Nymark-McMahon, M.H., Yieh, L., and Sandmeyer, S.B. (2001). Integrase 
mediates nuclear localization of Ty3. Mol Cell Biol 21, 7826-7838.

647 Llano, M., Saenz, D.T., Meehan, A., Wongthida, P., Peretz, M., Walker, W.H., Teo, W., and 648 Poeschla, E.M. (2006). An essential role for LEDGF/p75 in HIV integration. Science. 314, $649 \quad 461-464$.

650 Lusic, M., and Siliciano, R.F. (2017). Nuclear landscape of HIV-1 infection and integration. 651 Nat. Rev. Microbiol. 15, 69-82.

652 Manhas, S., Ma, L., and Measday, V. (2018). The yeast Ty1 retrotransposon requires 653 components of the nuclear pore complex for transcription and genomic integration. Nucleic 654 Acids Res. 46, 3552-3578.

655 Marini, B., Kertesz-Farkas, A., Ali, H., Lucic, B., Lisek, K., Manganaro, L., Pongor, S., 656 Luzzati, R., Recchia, A., Mavilio, F., et al. (2015). Nuclear architecture dictates HIV-1 657 integration site selection. Nature 521, 227-231.

658 Maskell, D.P., Renault, L., Serrao, E., Lesbats, P., Matadeen, R., Hare, S., Lindemann, D., 659 Engelman, A.N., Costa, A., and Cherepanov, P. (2015). Structural basis for retroviral 660 integration into nucleosomes. Nature 523, 366-369.

661 McLane, L.M., Pulliam, K.F., Devine, S.E., and Corbett, A.H. (2008). The Ty1 integrase 662 protein can exploit the classical nuclear protein import machinery for entry into the nucleus. 663 Nucleic Acids Res. 36, 4317-4326.

664 Michieletto, D., Lusic, M., Marenduzzo, D., and Orlandini, E. (2019). Physical principles of 665 retroviral integration in the human genome. Nat. Commun. 10, 575-587.

666 Molenberghs, F., Bogers, J.J., and De Vos, W.H. (2020). Confined no more: Viral 667 mechanisms of nuclear entry and egress. Int. J. Biochem. Cell Biol. 129, 105875.

668 Moore, S.P., Rinckel, L.A., and Garfinkel, D.J. (1998). A Ty1 Integrase Nuclear Localization 669 Signal Required for Retrotransposition. Mol Cell Biol 18, 1105-1114.

670 Morillon, A., Bénard, L., Springer, M., and Lesage, P. (2002). Differential Effects of 671 Chromatin and Gen4 on the 50-Fold Range of Expression among Individual Yeast Ty1 672 Retrotransposons. Mol Cell Biol 22, 2078-2088. 
673 Mularoni, L., Zhou, Y., Bowen, T., Gangadharan, S., Wheelan, S.J., and Boeke, J.D. (2012).

674 Retrotransposon Ty1 integration targets specifically positioned asymmetric nucleosomal 675 DNA segments in tRNA hotspots. Genome Res. 22, 693-703.

676 Neuvéglise, C., Feldmann, H., Bon, E., Gaillardin, C., and Casaregola, S. (2002). Genomic

677 evolution of the long terminal repeat retrotransposons in hemiascomycetous yeasts. Genome 678 Res. 12, 930-943.

679 Ocampo-Hafalla, M.T., and Uhlmann, F. (2011). Cohesin loading and sliding. J. Cell Sci. $680124,685-691$.

681 Oficjalska-Pham, D., Harismendy, O., Smagowicz, W.J., Gonzalez de Peredo, A., Boguta, 682 M., Sentenac, A., and Lefebvre, O. (2006). General repression of RNA polymerase III 683 transcription is triggered by protein phosphatase type 2A-mediated dephosphorylation of 684 Maf1. Mol Cell 22, 623-632.

685 Patterson, K., Shavarebi, F., Magnan, C., Chang, I., Qi, X., Baldi, P., Bilanchone, V., and 686 Sandmeyer, S.B. (2019). Local features determine Ty3 targeting frequency at RNA 687 polymerase III transcription start sites. Genome Res. 29, 1298-1309.

688 Patterson, M.N., Scannapieco, A.E., Au, P.H., Dorsey, S., Royer, C. a., and Maxwell, P.H. 689 (2015). Preferential retrotransposition in aging yeast mother cells is correlated with increased 690 genome instability. DNA Repair (Amst). 34, 18-27.

691 Qi, X., and Sandmeyer, S. (2012). In vitro targeting of strand transfer by the Ty3 retroelement 692 integrase. J Biol Chem 287, 18589-18595.

693 Qi, X., Daily, K., Nguyen, K., Wang, H., Mayhew, D., Rigor, P., Forouzan, S., Johnston, M., 694 Mitra, R.D., Baldi, P., et al. (2012). Retrotransposon profiling of RNA polymerase III 695 initiation sites. Genome Res 22, 681-692.

696 Quadrana, L., Etcheverry, M., Gilly, A., Caillieux, E., Madoui, M.A., Guy, J., Bortolini 697 Silveira, A., Engelen, S., Baillet, V., Wincker, P., et al. (2019). Transposition favors the 698 generation of large effect mutations that may facilitate rapid adaption. Nat. Commun. 10, $699 \quad 3421-3430$.

700 De Rijck, J., de Kogel, C., Demeulemeester, J., Vets, S., El Ashkar, S., Malani, N., Bushman, 
701 F.D., Landuyt, B., Husson, S.J., Busschots, K., et al. (2013). The BET Family of Proteins

702 Targets Moloney Murine Leukemia Virus Integration near Transcription Start Sites. Cell Rep.

$7035,886-894$.

704 Risler, J.K., Kenny, A.E., Palumbo, R.J., Gamache, E.R., and Curcio, M.J. (2012). Host co705 factors of the retrovirus-like transposon Ty1. Mob. DNA 3, 12.

706 Rothenbusch, U., Sawatzki, M., Chang, Y., Caesar, S., and Schlenstedt, G. (2012).

707 Sumoylation regulates Kap114-mediated nuclear transport. EMBO J. 31, 2461-2472.

708 Sharma, A., Larue, R.C., Plumb, M.R., Malani, N., Male, F., Slaughter, A., Kessl, J.J., 709 Shkriabai, N., Coward, E., Aiyer, S.S., et al. (2013). BET proteins promote efficient murine 710 leukemia virus integration at transcription start sites. Proc. Natl. Acad. Sci. U. S. A. 110, $711 \quad 12036-12041$.

712 Sharma, D., De Falco, L., Padavattan, S., Rao, C., Geifman-Shochat, S., Liu, C.F., and 713 Davey, C.A. (2019). PARP1 exhibits enhanced association and catalytic efficiency with $714 \quad \gamma$ H2A.X-nucleosome. Nat. Commun. 10, 5751-5763.

715 Souciet, J.L., Dujon, B., Gaillardin, C., Johnston, M., Baret, P. V., Cliften, P., Sherman, D.J., 716 Weissenbach, J., Westhof, E., Wincker, P., et al. (2009). Comparative genomics of protoploid 717 Saccharomycetaceae. Genome Res. 19, 1696-1709.

718 Spaller, T., Kling, E., Glöckner, G., Hillmann, F., and Winckler, T. (2016). Convergent 719 evolution of tRNA gene targeting preferences in compact genomes. Mob. DNA 7, 17.

720 Sultana, T., Zamborlini, A., Cristofari, G., and Lesage, P. (2017). Integration site selection by 721 retroviruses and transposable elements in eukaryotes. Nat. Rev. Genet. 18, 292-308.

722 Szilard, R.K., Jacques, P.E., Laramée, L., Cheng, B., Galicia, S., Bataille, A.R., Yeung, M., 723 Mendez, M., Bergeron, M., Robert, F., et al. (2011). Systematic identification of fragile sites 724 via genome-wide location analysis of $\gamma$-H2AX. Nat. Struct. Mol. Biol. 17, 299-305.

725 Vannini, A., and Cramer, P. (2012). Conservation between the RNA polymerase I, II, and III 726 transcription initiation machineries. Mol. Cell 45, 439-446.

727 Vorländer, M.K., Khatter, H., Wetzel, R., Hagen, W.J.H., and Müller, C.W. (2018).

728 Molecular mechanism of promoter opening by RNA polymerase III. Nature 553, 295-300. 
729 Wilson, M.D., Renault, L., Maskell, D.P., Ghoneim, M., Pye, V.E., Nans, A., Rueda, D.S.,

730 Cherepanov, P., and Costa, A. (2019). Retroviral integration into nucleosomes through DNA

731 looping and sliding along the histone octamer. Nat. Commun. 10, 4189-4198.

732 Wu, C.C., Lin, Y.C., and Chen, H.T. (2011). The TFIIF-like Rpc37/53 dimer lies at the center

733 of a protein network to connect TFIIIC, Bdp1, and the RNA polymerase III active center. Mol

734 Cell Biol 31, 2715-2728.

735 Xie, W., Gai, X., Zhu, Y., Zappulla, D.C., Sternglanz, R., and Voytas, D.F. (2001). Targeting

736 of the yeast Ty5 retrotransposon to silent chromatin is mediated by interactions between

737 integrase and Sir4p. Mol Cell Biol 21, 6606-6614.

738 Yieh, L., Kassavetis, G., Geiduschek, E.P., and Sandmeyer, S.B. (2000). The Brf and TATA-

739 binding protein subunits of the RNA polymerase III transcription factor IIIB mediate

740 position-specific integration of the gypsy-like element, Ty3. J Biol Chem 275, 29800-29807.

741 Yieh, L., Hatzis, H., Kassavetis, G., and Sandmeyer, S.B. (2002). Mutational analysis of the

742 transcription factor IIIB-DNA target of Ty3 retroelement integration. J Biol Chem 277,

$743 \quad 25920-25928$.

744 Zhu, Y., Dai, J., Fuerst, P.G., and Voytas, D.F. (2003). From the Cover: Controlling

745 integration specificity of a yeast retrotransposon. Proc Natl Acad Sci U S A 100, 5891-5895.

746 Zou, S., Ke, N., Kim, J.M., and Voytas, D.F. (1996). The Saccharomyces Retrotransposon

747 Ty5 Integrates Preferentially into Regions of Silent Chromatin at the Telomeres and Mating

748 Loci. Genes Dev 10, 634-645. 
752 Figure 1. Site selection and regulation of the integration of Ty1, Ty3 and Ty5 LTR753 retrotransposons. Ty1 retrotransposon integrates in a $1 \mathrm{~kb}$-window upstream of Pol III754 transcribed genes and can be retargeted into subtelomeres as secondary targets. Ty 3 integrates 755 in a narrower window of $20 \mathrm{bp}$ upstream of Pol III-transcribed genes. Ty5 integrates into 756 heterochromatin of telomeres and silent mating type loci HML/HMR. The main drivers of 757 Ty1, Ty3 and Ty5 integration selectivity have been clearly identified as the RNA Pol III 758 subunit AC40, TFIIIB and Sir4, respectively. Other factors have also been suggested as 759 influencing Ty integration pattern although many shadow areas remain to be clarified.

\section{Figure 2.}

762 A. The Nuclear Pore Complex has a central role in the replication cycle of Ty LTR763 retrotransposons. Schematic representation of the nuclear pore complex listing individual members of each structural part. The nucleoporins identified in large-scale screens as regulating Ty1 and Ty3 retrotransposition rate are indicated in bold (Aye et al., 2004; Dakshinamurthy et al., 2010; Griffith et al., 2003; Irwin et al., 2005; Risler et al., 2012). The nucleoporins for which mutation or deletion triggers a decrease in Ty1 integration upstream of two $t D N A$ s without affecting Tyl overall integration frequency (Manhas et al., 2018), are underlined.

B. Coupling the nuclear import of Ty1 IN:cDNA complex and Ty1 integration at Pol III transcribed genes. The presence of the targeting domain interacting with AC40 within the unusually long linker of the bipartite NLS of Ty1 integrase opens the possibility of coupling between pre-integration complex nuclear import and integration targeting. The binding of the two basic amino acid-rich regions of IN1 bNLS to the two NLS-binding pockets of importin$\alpha$ could expose the targeting sequence to AC40. Once in the nucleus, the interaction with AC40 would help Ran-GTP to dissociate the IN1-importin- $\alpha / \beta$ complex. This coupling between nuclear entry and Pol III interaction would also be reinforced by the recruitment of $t D N A s$ to the nuclear pore to be transcribed. 


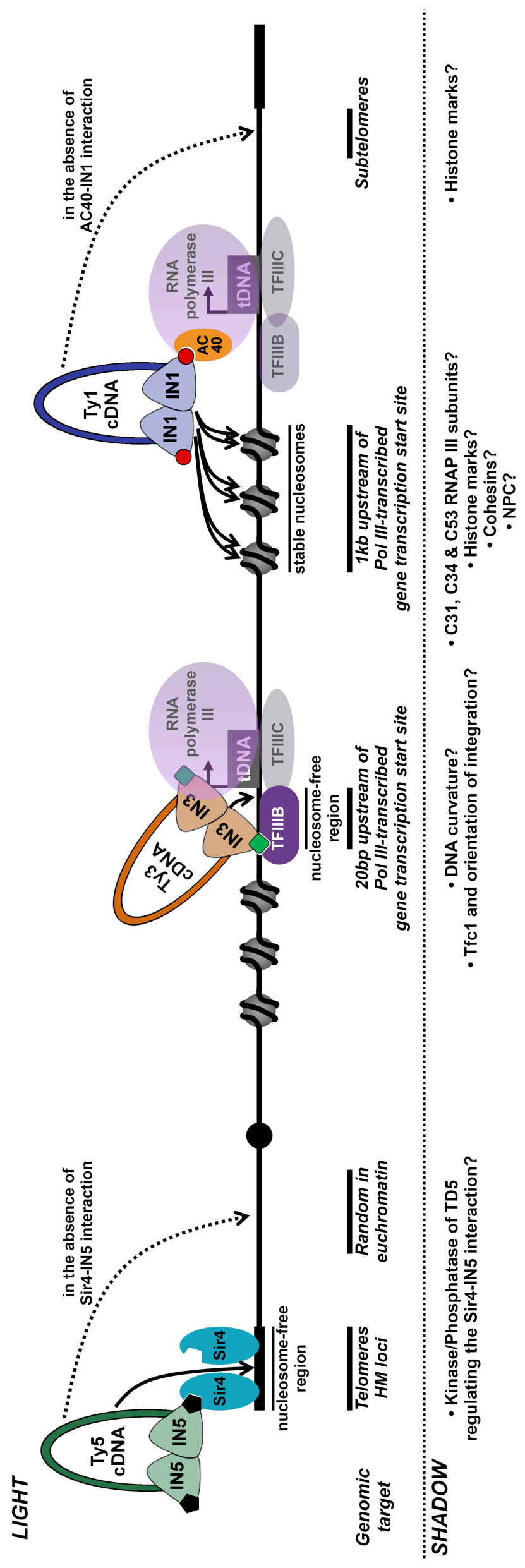


a

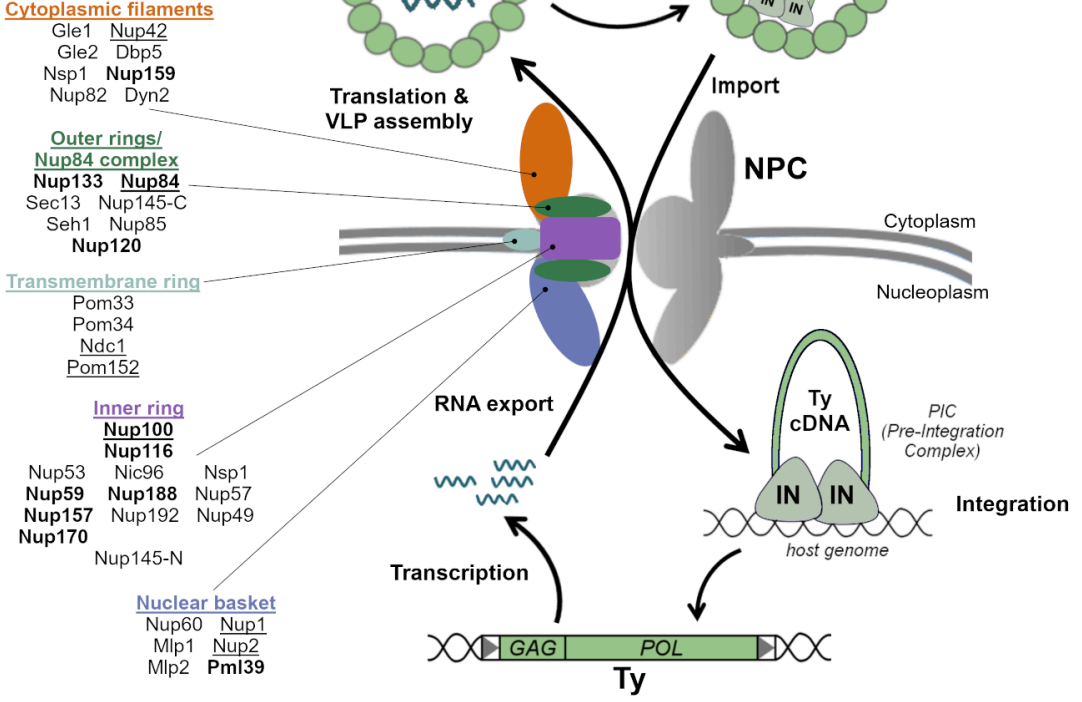

b

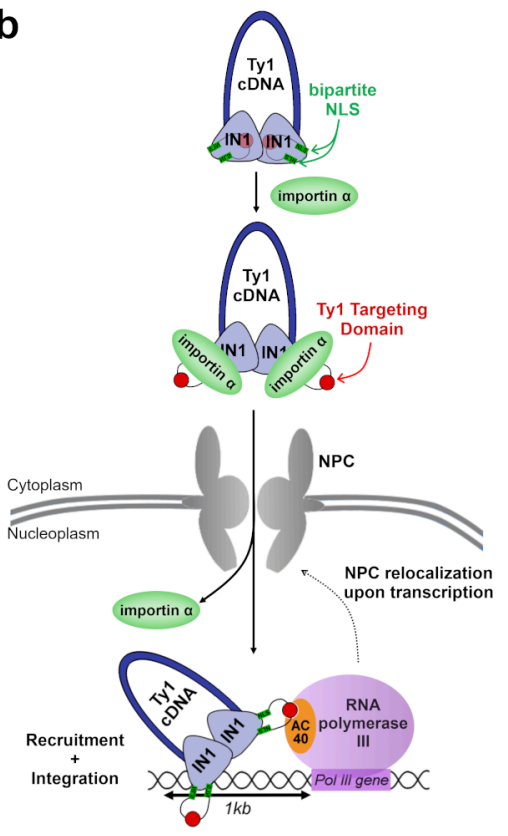

